\title{
Relation of Critical Current and $n$-Value of Local Sections to Those of Sample in a Multi-Filamentary Bi2223 Superconducting Composite Tape with Applied Stress-Induced Cracks
}

\author{
Shojiro Ochiai ${ }^{1}$, Hiroshi Okuda ${ }^{2}$, Hiroshi Matsubayashi ${ }^{2, *}$, Kozo Osamura $^{3}$ and Alex Otto ${ }^{4}$ \\ ${ }^{1}$ Elements Strategy Initiative for Structural Materials, Kyoto University, Kyoto 606-8501, Japan \\ ${ }^{2}$ Department of Materials Science and Engineering, Kyoto University, Kyoto 606-8501, Japan \\ ${ }^{3}$ Research Institute for Applied Sciences, Kyoto 606-8202, Japan \\ ${ }^{4}$ American Superconductor Corporations, 2 Technology Drive, Westborough, MA 01581, USA
}

Under the application of tensile stress to a multi-filamentary $\mathrm{Bi}_{2} \mathrm{Sr}_{2} \mathrm{Ca}_{2} \mathrm{Cu}_{3} \mathrm{O}_{10+\mathrm{x}}(\mathrm{Bi} 2223)$ composite tape sample composed of a series of sections, the relation of superconducting properties (voltage-current curve, critical current and $n$-value) of sections to those of the sample were studied experimentally and analytically. The voltage-current curve of the most extensively cracked section whose critical current was the lowest among the sections, was the nearest in position to the voltage-current curve of the sample and played a dominant role in determination of the critical current of the sample. On the other hand, the $n$-value of the sample was significantly affected not only by the voltage-current curve of the most extensively cracked section but also on the position in relation to the voltage- current curves between the most extensively cracked section and the other sections. The denser the voltage-current curve of the other sections in the neighborhood of the voltage-current curve of the most extensively cracked section, the higher the $n$-value of sample became. In analysis of the experimental results, an equivalent crack current shunting model, in which multiple cracks existing inside were replaced by an equivalent crack, was employed. With this model, the measured critical current and $n$-value of both sections and sample, and the correlation between $n$-value and critical current both for sections and sample, were described satisfactorily. [doi:10.2320/matertrans.MBW201502]

(Received September 29, 2015; Accepted February 22, 2016; Published April 1, 2016)

Keywords: Bi2223 superconducting tape, sample length, crack, critical current, $n$-value

\section{Introduction}

Thermal, mechanical and electromagnetic stresses are exerted to superconducting coated- and filamentary- composite tapes during fabrication and in service. At high stresses, the superconducting layers in coated conductors are cracked and, as a result, the critical current $I_{\mathrm{c}}$ and $n$-value are seriously reduced, as has been shown for $\mathrm{YBCO}^{1,2)}$, $\mathrm{DyBCO}^{3-6)}, \mathrm{SmB}$ $\mathrm{CO}^{7,8)}$ and $\mathrm{GdBCO}^{9)}$ conductors. The same situation occurs also in the filamentary $\mathrm{Bi}_{2} \mathrm{Sr}_{2} \mathrm{Ca}_{2} \mathrm{Cu}_{3} \mathrm{O}_{10+x}(\mathrm{Bi} 2223)^{10-12)}$, $\mathrm{Nb}_{3} \mathrm{Sn}^{13)}, \mathrm{Nb}_{3} \mathrm{Al}^{14)}$ and $\mathrm{MgB}_{2}{ }^{15,16)}$ conductors due to the formation of collective cracks (cracks composed of successively cracked filaments in a transverse cross-section ${ }^{12,13)}$ ). Cracking of superconducting layer/filament takes place heterogeneously within a sample $e^{8,12,14)}$. Accordingly, in a sample constituted of a series of local sections, the $I_{\mathrm{c}}$ and $n$-value vary from section to section. It is required to clarify the influence of extent of cracking on the $I_{\mathrm{c}}$ and $n$-value of sections and sample and also the relation of the $I_{\mathrm{c}}$ and $n$-value of sections to those of sample.

The present work was carried out to reveal the correlation of the $I_{\mathrm{c}}$ and $n$-value of sections to that of sample in wide range of crack evolution. The Bi2223 filamentary conductor tape was used for test. The configuration of the sample for test is shown in Fig. 1, in which the sections ( $\mathrm{S} 1$ to S6) were arrayed as a series in the direction of the sample length. As the electric circuit of a sample was composed of series circuits of sections, we could detect experimentally and also could estimate analytically how the superconducting property of the sample varied, depending on the difference in extent of cracking among the sections.

*Graduate Student, Kyoto University

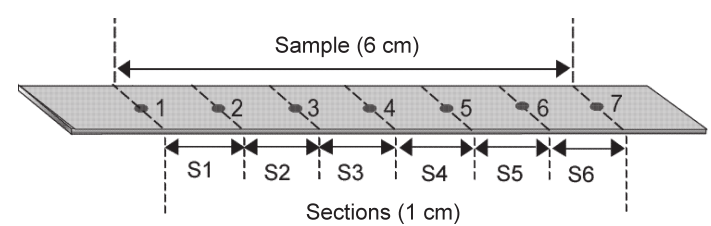

Fig. 1 Schematic representation of the test sample with a length $L=6 \mathrm{~cm}$, composed of six sections (S1 to S6) with a length $L=1 \mathrm{~cm}$. The numbers 1 to 7 refer to the location of the voltage taps. The $V-I$ curve, critical current $I_{\mathrm{c}}$ and $n$-value were measured for each section by using the voltage taps of $i$ and $i+1$ ( $i=1$ to 6 ) and sample by using the voltage tap of 1 and 7 .

In this work, a simple and practically useful method $3,4,8,17)$ is used for description of the relation between $I_{\mathrm{c}}$ and $n$-value. It is noted that this method is employed under the condition where $n$-value of sections and sample in non-cracked state could be approximated roughly as constant below electric field $10 \mu \mathrm{V} / \mathrm{cm}$. This condition is satisfied in this work, whose details will be shown later in Section 3. When the condition is satisfied, the present approach can be utilized for description of $I_{\mathrm{c}}$ and $n$-value under heterogeneous crack evolution in both filamentary- and coated- conductor tapes.

\section{Experimental Procedure}

The high critical current density type multi-filamentary Bi2223 composite tape ${ }^{18)}$, consisting of 55 Bi2223 filaments, Ag (stabilizer) and Ag-Li alloy (sheath), fabricated at American Superconductor Corporation, was used for study, as in our former works to study the influence of applied tensile ${ }^{12)}$ and bending ${ }^{19)}$-strains on $I_{\mathrm{c}}$ and $n$-value. The width and thickness of the tape were 4.1 and $0.22 \mathrm{~mm}$, respectively. For test, 
we prepared the samples with a length $L=6 \mathrm{~cm}$, constituting of six local sections with a length $L=1 \mathrm{~cm}$, as has been shown in Fig. 1. In experiment, seven voltage taps ("1" to "7") were attached to the tape surface with a distance of $L=1 \mathrm{~cm}$ between each of them. The $V$ (voltage) $-I$ (current) curve was measured for each section by using the voltage taps of " $i$ " and " $i+1$ " ( $i=1$ to 6$)$ and for sample by using the voltage taps of " 1 " and " 7 ". Due to the heterogeneous cracking behavior, the strains of sections were different to each other but stress was common for all sections and sample. Due to this reason, stress was used as a measure of applied mechanical condition, instead of strain.

The $V-I$ curves of 6 sections ( $\mathrm{S} 1$ to S6) and sample were measured under applied tensile stress $\sigma_{\mathrm{T}}$ at $77 \mathrm{~K}$ in a self-magnetic field. Three samples (hereafter, noted as Sample 1, Sample 2 and Sample 3) with a configuration shown in Fig. 1 were used in experiment. The critical current $I_{\mathrm{c}}$ was estimated with an electric field criterion of $E_{\mathrm{c}}=1 \mu \mathrm{V} / \mathrm{cm}$, where electric filed $E$ is defined by $V / L$. The critical electric field $E_{\mathrm{c}}=1 \mu \mathrm{V} / \mathrm{cm}$ corresponds to a critical voltage $V_{\mathrm{c}}=$ $L E_{\mathrm{c}}=1 \mu \mathrm{V}$ for sections with a length of $L=1 \mathrm{~cm}$ and to $V_{\mathrm{c}}=$ $6 \mu \mathrm{V}$ for samples with a length of $L=6 \mathrm{~cm}$. The $n$-value was defined as the index of the form $E \propto I^{n}$ in the electric range of $E=V / L=0.1 \sim 10 \mu \mathrm{V} / \mathrm{cm}$. It was estimated by fitting the measured $V-I$ curve to the form $V \propto I^{n}$ in the voltage range of $V=$ $0.1 \sim 10 \mu \mathrm{V}$ for sections and $V=0.6 \sim 60 \mu \mathrm{V}$ for sample.

\section{Results and Discussion}

\subsection{Measured $V$ (voltage) $-I$ (current) curves, $I_{\mathrm{c}}$ (critical current)-values and $\boldsymbol{n}$-values of sections and samples}

Figure 2 shows the measured $V-I$ curves of samples and their sections (S1 to S6) under applied tensile stress $\sigma_{\mathrm{T}}$, in which the experimental results of (a e) Sample 1 and (a' e') Sample 3 are representatively presented. From each $V-I$ curve, $I_{\mathrm{c}^{-}}$and $n$-values for each section and for each sample were obtained with the procedure stated in Section 2. Figure $3(\mathrm{a}, \mathrm{b}, \mathrm{c})$ show the change in $I_{\mathrm{c}}-$ value and (a', b', c') the change in $n$-value with increasing $\sigma_{\mathrm{T}}$ for Samples 1, 2 and 3 and their sections (S1 to S6). The following features are read from Figs. 2 and 3.

(1) Cracking of filaments and hence reduction in $I_{\mathrm{c}}$ and $n$-value took place for $\sigma_{\mathrm{T}}>78 \mathrm{MPa}$ in all samples, as shown in Fig. 3 where the boundary between the non-cracked and
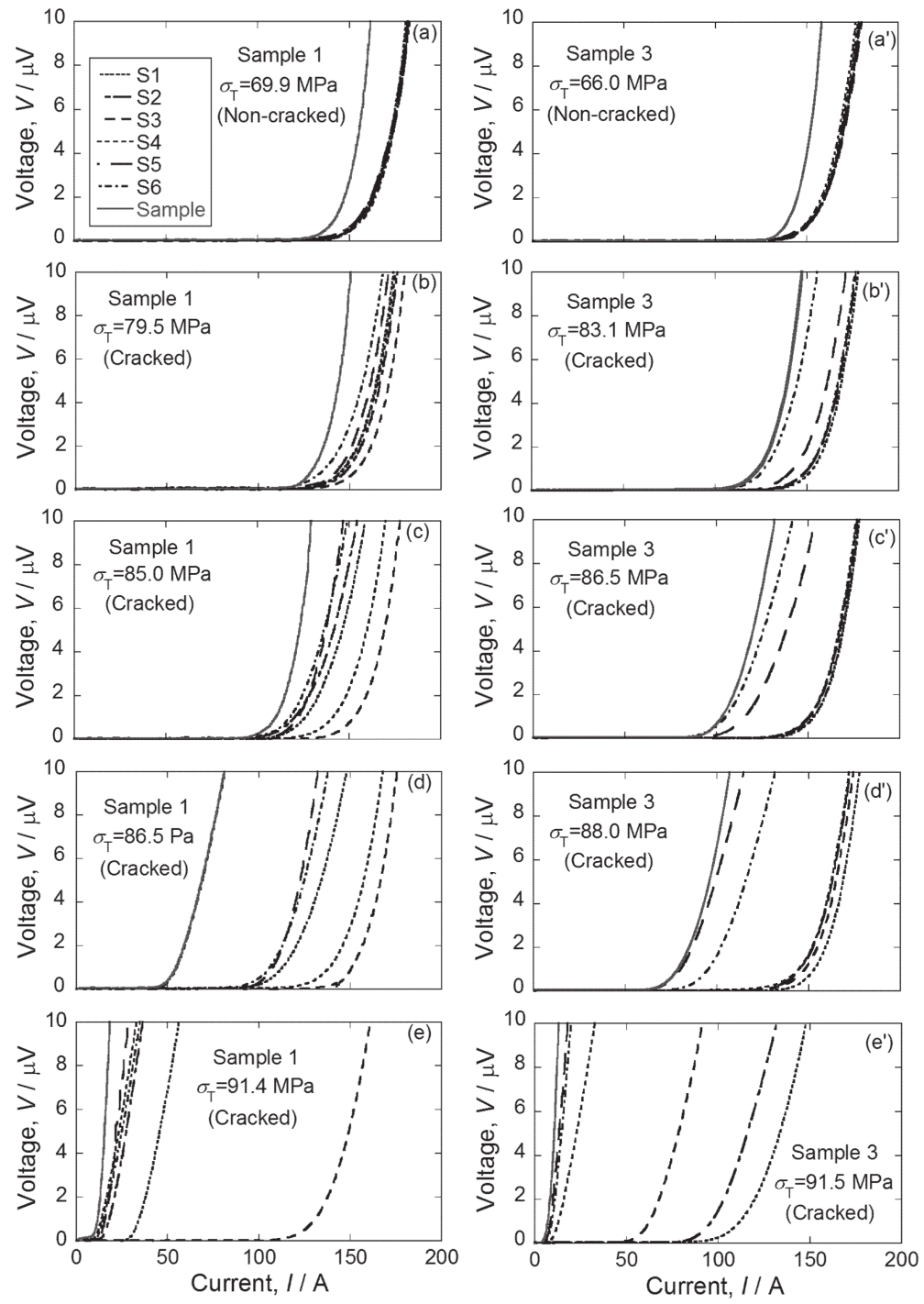

Fig. 2 Representative $V$ (voltage) $-I$ (current) curves of the sections (S1 to S6) and samples. (a) to (e) and (a') to (e') show the results of Sample 1 and Sample 3, respectively. 

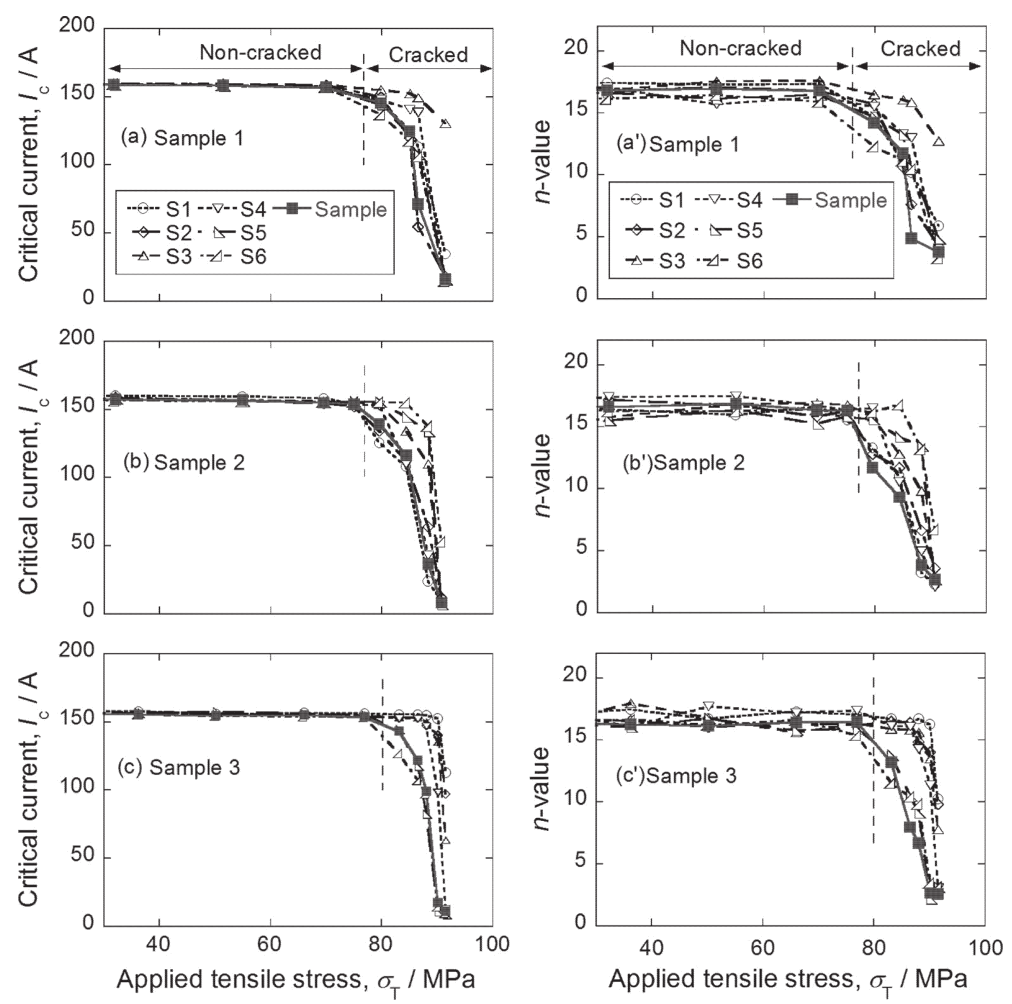

Fig. 3 Change in (a, b, c) critical current $I_{\mathrm{c}}$ and (a', b', c') $n$-value of the sections (S1 to S6) and overall sample with increasing applied stress $\sigma_{\mathrm{T}}$. (a, a'), (b, $\left.b^{\prime}\right)$ and (c, c') show the experimental results of Samples 1, 2 and 3, respectively.

cracked regions is indicated with broken line. In the cracked region, both of $I_{\mathrm{c}^{-}}$and $n$-values decreased significantly with increasing $\sigma_{\mathrm{T}}$ due to enhancement of crack evolution. The extent of decrease in $I_{\mathrm{c}^{-}}$and $n$-values with $\sigma_{\mathrm{T}}$ was different from section to section and from sample to sample, due to the heterogeneous crack evolution.

(2) In Fig. 2 (a, a') showing the $V-I$ curves in non-cracked region, the location and shape of the $V-I$ curves of sections were almost the same. Accordingly, the difference in $I_{\mathrm{c}}$-value and the difference in $n$-value among the sections were very small before the onset of cracking (Fig. 3). On the other hand, once cracking took place, the $V-I$ curves of the sections (Fig. 2 (b e), (b' e')) shifted to lower current range. The shift of the $V-I$ curves became dominant with increasing $\sigma_{\mathrm{T}}$, resulting in more reduction both in $I_{\mathrm{c}}$ and $n$-value at higher $\sigma_{\mathrm{T}}$ (Fig. 3).

(3) The $I_{\mathrm{c}}$-value of sample was slightly higher than the lowest $I_{\mathrm{c}}$-value of the most extensively cracked section and was lower than the value of less cracked sections (Fig. 3(a, b, c)); namely the $I_{\mathrm{c}}$-value of samples was in the range between the lowest and highest $I_{\mathrm{c}}$-values of the sections. On the other hand, while $n$-value of sample was in the range between the lowest and highest $n$-values of sections when the $V-I$ curve of sample was constructed by the $V-I$ curves of all or many sections, it became lower than the lowest $n$-value among sections when the $V-I$ curve of sample was constructed by the $V-I$ curves of one or a few sections (Fig. 3(a', b', c')).

\subsection{Model of crack-induced current shunting}

The following features have been known for the cracking behavior of multi-filamentary composite tape under applied tensile stress. (i) The extent of cracking varies along the tape length and the extent of cracking differs among the sections ${ }^{12,20)}$. This feature was reconfirmed in Figs. 2 and 3 in the present work. (ii) Collective cracks (cracks composed of successively cracked filaments in a transverse cross-section ${ }^{12,13)}$ ) are formed multiply due to the stress concentration-induced successive fracture of filaments ${ }^{21-23)}$.

According to the partial crack-current shunting model of Fang et al. ${ }^{24)}$, (a) current path and (b) simplified electrical circuit under an existent collective crack are schematically shown in Fig. $4^{17)}$. In our preceding works for study on $I_{\mathrm{c}^{-}}$and $n$-values of coated conductors in which the superconducting layer was multiply cracked ${ }^{3,4,8,17)}$, we replaced the multiple cracks by a single equivalent crack in application of the model, since the current shunting occurs in the same mechanism in both single and multiple cracks. The experimental results were described well despite the replacement, and it was shown that such a model approach is a useful tool in practice for analysis ${ }^{3,4,8,17)}$. Then, for study on $I_{\mathrm{c}^{-}}$and $n$-values of cracked Bi2223 filamentary conductor ${ }^{12,17)}$, we replaced the Bi2223 multi-filaments in the tape by a single equivalent filament and also we replaced the collective multiple cracks by a single equivalent partial crack. It was found that such an equivalent crack-current shunting model approach is a useful tool also for analysis of filamentary conductor ${ }^{12,17)}$. In the present work, this approach was used for analysis of the experimental results. The outline of this approach is shown below.

In the transverse cross-section in Fig. 4 in which a partial crack exists, the cracked part corresponds to an equivalent collective crack that has lost superconductivity and the ligament part corresponds to a surviving part that keeps superconductivity. The ratio of cross-sectional area of cracked part 

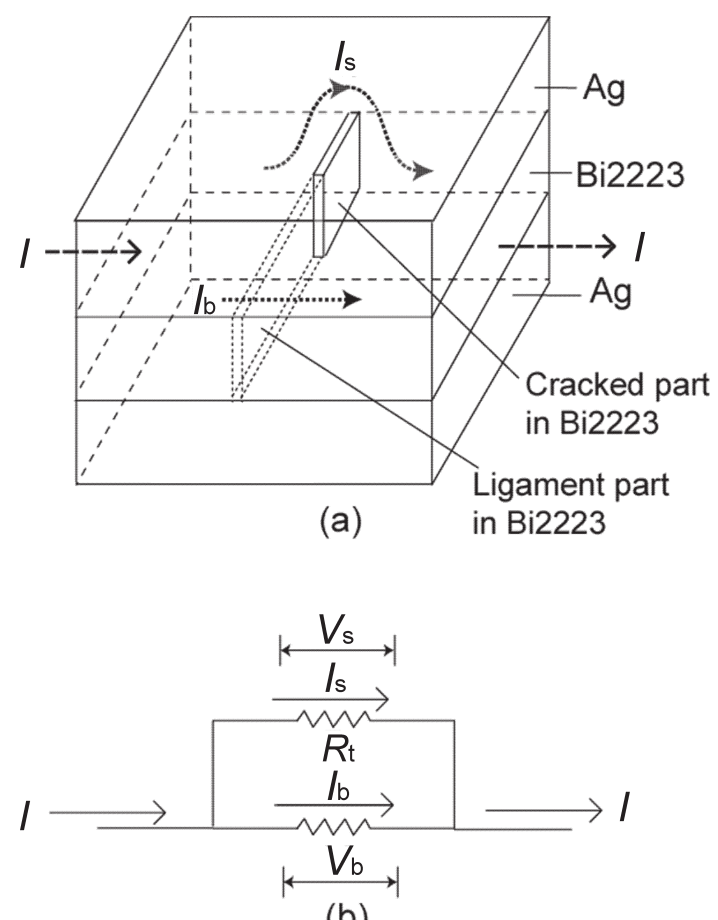

(b)

Fig. 4 Schematic representation of (a) current path and (b) simplified electrical circuit under an existent partial $\mathrm{crack}^{17)}$.

to the total cross-sectional area of the filament is defined as $f$. The ligament part with an area ratio $1-f$ transports current $I_{\mathrm{b}}$. At the cracked part with an area ratio $f$, current $I_{\mathrm{S}}\left(=I-I_{\mathrm{b}}\right)$ shunts into Ag. In the shunting circuit, the resistance at the Bi2223-Ag interface and resistance in Ag are included in the total resistance $R_{\mathrm{t}}$ (Fig. 4(b)). The voltage, developed in the ligament part that transports current $I_{\mathrm{b}}$, is noted as $V_{\mathrm{b}}$ in Fig. 4(b). The voltage $V_{\mathrm{s}}=I_{\mathrm{S}} R_{\mathrm{t}}$, developed by shunting current $I_{\mathrm{s}}$ in the cracked part, is equal to $V_{\mathrm{b}}$ since the non-cracked and cracked parts constitute of a parallel circuit.

The relation of voltage $V$ to current $I$ in non-cracked state is expressed as

$$
V=E_{\mathrm{c}} L\left(\frac{I}{I_{\mathrm{c} 0}}\right)^{n_{0}}
$$

where $I_{\mathrm{c} 0}$ and $n_{0}$ are the critical current and $n$-value in the original non-cracked state, respectively, $E_{\mathrm{c}}$ is the critical electric field for determination of critical current $(=1 \mu \mathrm{V} / \mathrm{cm})$ and $L$ is the voltage probe distance $(=1 \mathrm{~cm}$ for sections and $=$ $6 \mathrm{~cm}$ for samples). When the sample is very long, $n$-value shall be smaller at higher field criterion due to the transition from superconducting- to normal conducting state even in non-cracked state. However, in the experimental result of the present sample and sections, such a transition was not found in the non-cracked state. Accordingly, the $n$-value of sample was comparable with the $n$-values of sections, as shown in Fig. 3. Due to this result, the $V-I$ relation in non-cracked state was given by eq. (1) commonly for sample and sections.

In the cracked state, the voltage $V$ is the sum of the voltage $E_{c} L\left(I / I_{\mathrm{c} 0}\right)^{n_{0}}$ developed at the non-cracked area apart from the cracked cross-section and the voltage $V_{\mathrm{b}}$ developed at the cracked part. Noting the current transfer length as $\mathrm{s}(\ll L)^{5,6)}$ and modifying the formulations of Fang et al. ${ }^{24)}$, we have the constitutional equations for $V-I$ curve in the form ${ }^{3-6,8,12,17)}$;

$$
\begin{gathered}
V=E_{\mathrm{c}} L\left(\frac{I}{I_{\mathrm{c} 0}}\right)^{n_{0}}+V_{\mathrm{b}} \\
I=I_{\mathrm{b}}+I_{\mathrm{s}}=I_{\mathrm{c} 0}(1-f)\left(\frac{L}{s}\right)^{1 / n_{0}}\left[\frac{V_{\mathrm{b}}}{E_{\mathrm{c}} L}\right]^{1 / n_{0}}+\frac{V_{\mathrm{b}}}{R_{\mathrm{t}}}
\end{gathered}
$$

The term $(1-f)(L / s)^{1 / n_{0}}$, hereafter noted as a ligament parameter, has the following physical meaning. (1) It refers to the size of the ligament and hence it can be used as a measure of size of ligament and crack; the larger the $(1-f)(L / s)^{1 / n_{0}}$, the larger is the ligament and hence the smaller is the crack. (2) When the $(1-f)(L / s)^{1 / n_{0}}$ is small, namely when the crack is large, the voltage $V_{\mathrm{b}}$ developed at the cracked part is far higher than the voltage $E_{\mathrm{c}} L\left(I / I_{\mathrm{c} 0}\right)^{n_{0}}$ and accordingly $V=V_{\mathrm{b}}$ is held $^{3,4,8,12)}$. In such a situation, setting $E_{\mathrm{c}} L\left(I / I_{\mathrm{c} 0}\right)^{n_{0}}=0$ and $V_{\mathrm{b}}=V$ in eqs. (2) and (3), we have the $V-I$ relation in one equation:

$$
I=I_{\mathrm{b}}+I_{\mathrm{s}}=I_{\mathrm{c} 0}(1-f)\left(\frac{L}{s}\right)^{1 / n_{0}}\left[\frac{V}{E_{\mathrm{c}} L}\right]^{1 / n_{0}}+\frac{V}{R_{\mathrm{t}}}
$$

Critical current $I_{\mathrm{c}}$ at $V=V_{\mathrm{c}}=E_{\mathrm{c}} L$ is expressed by

$$
I=I_{\mathrm{c} 0}(1-f)\left(\frac{L}{s}\right)^{1 / n_{0}}+\frac{V_{\mathrm{c}}}{R_{\mathrm{t}}}
$$

The shunting current $I_{\mathrm{S}}$ at $V=V_{\mathrm{c}}$ is given by $V_{\mathrm{c}} / R_{\mathrm{t}}$. If we take a virtual case where $R_{\mathrm{t}}$ is high and shunting current $I_{\mathrm{S}}$ is negligible in comparison with the term $I_{\mathrm{c} 0}(1-f)(L / s)^{1 / n_{0}}$ in eq. (5), $I_{\mathrm{c}} / I_{\mathrm{c} 0}$ is expressed as $I_{\mathrm{c}} / I_{\mathrm{c} 0}=(1-f)(L / s)^{1 / n_{0}}$. This means that the ligament parameter $(1-f)(L / s)^{1 / n_{0}}$ has a physical meaning as $I_{\mathrm{c}} / I_{\mathrm{c} 0}$ (ratio of the critical current $I_{\mathrm{c}}$ in cracked state to the critical current $I_{\mathrm{c} 0}$ in non-cracked state) in an virtual case where voltage is developed only at the crack and also no shunting current takes place ${ }^{5,6)}$.

The term $(1-f)(L / s)^{1 / n_{0}}$ is equal to $I_{\mathrm{c}} / I_{\mathrm{c} 0}$ only under the virtual conditions stated above. In practical tapes, current shunting takes place, and accordingly, $(1-f)(L / s)^{1 / n_{0}}$ is not equal to $I_{\mathrm{c}} / I_{\mathrm{c} 0}$. However, as the shunting current is low at low voltage such as $V_{\mathrm{c}}^{3,4,12)}$, the ligament parameter can give fairly a good first approximation for $I_{\mathrm{c}} / I_{\mathrm{c} 0}$ in both sections and samples (details are shown later in Subsection 3.4). The values of $(1-f)(L / s)^{1 / n_{0}}$ and $R_{\mathrm{t}}$ can be estimated by fitting the measured $V-I$ curves to eqs. (2) and (3) without estimation of the values of $1-f$ and $s$, separately. Once they are estimated, the Bi2223 ligament-transported current $I_{\mathrm{b}} \quad\left(=I_{\mathrm{c} 0}(1-\right.$ $\left.f)(L / s)^{1 / n_{0}}\left\{V_{\mathrm{b}} /\left(E_{\mathrm{c}} L\right)\right\}^{1 / n_{0}}\right)$ and shunting current $I_{\mathrm{s}}\left(=V_{\mathrm{b}} / R_{\mathrm{t}}\right)$ in the partially cracked cross-section can be obtained ${ }^{3,4,8,12,17)}$.

\subsection{Reproducibility of the measured $I_{\mathrm{c}^{-}}$and $n$-values of sections and samples with an equivalent crack-cur- rent shunting model}

The values of $(1-f)(L / s)^{1 / n_{0}}$ and $R_{\mathrm{t}}$ in eqs. (2) and (3) of sections and samples in the cracked region $\left(\sigma_{\mathrm{T}}>78 \mathrm{MPa}\right)$ were obtained, as shown in Fig. 5. The ligament parameter $(1-f)(L / s)^{1 / n_{0}}$ had the following features (Fig. 5(a, b, c)). (a) It decreased with increasing applied stress in both sections and sample, reflecting the crack extension with stress. (b) The ligament parameter value of sample was within the range of the highest and lowest values among the sections in all Samples 1,2 and 3. 

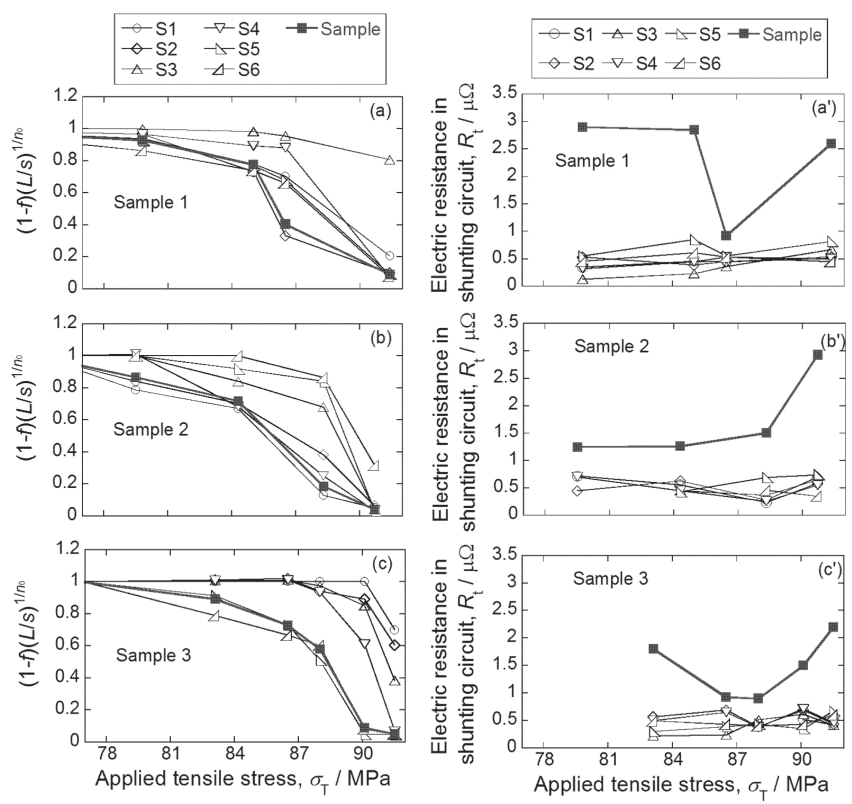

Fig. 5 Obtained values of $(\mathrm{a}, \mathrm{b}, \mathrm{c})(1-f)(L / s)^{1 / n_{0}}$ and (a', b', c') $R_{\mathrm{t}}$ in the sections S1 to S6 and Samples 1, 2 and 3, plotted against applied tensile stress $\sigma_{\mathrm{T}}$ higher than $78 \mathrm{MPa}$ where samples were cracked.

The $R_{\mathrm{t}}$ had the following features. (a) In some case, $R_{\mathrm{t}}$ increased with stress but in some case it decreased. Also in some case, $R_{\mathrm{t}}$ increased and then decreased. In this way, the change in $R_{\mathrm{t}}$ with stress was different among sections and among samples, reflecting the difference in crack evolution. (b) $R_{\mathrm{t}}$ of samples was larger than that of sections, reflecting that the sample was constituted of a series of sections. Also it is noted that when the $V-I$ curves of the sections were rather close to each other (Fig. 2(b) and (c)) or when the $V-I$ curves of plural sections were close to the $V-I$ curve of the most extensively cracked section (Fig. 2(e) and (e')), all or many sections contributed to the construction of the $V-I$ curve of sample. In such a case, $R_{\mathrm{t}}$ of the sample was high (Fig. 5(a', $\left.b^{\prime}, c^{\prime}\right)$ ). On the other hand, when only one or two far extensively cracked sections contributed to the construction of the $V-I$ curve of sample (Fig. 2(d), (c') and (d')), $R_{\mathrm{t}}$ of the sample was low (Fig. 5(a', b', c')). In this way, $R_{\mathrm{t}}$-value of the sample was significantly affected by the position relation of $V-I$ curve among the sections. How the $R_{\mathrm{t}}-$ value and also $I_{\mathrm{c}-}$ and $n$-values of sample are affected by the position relation of $V-I$ curve among sections will be discussed in detail in Subsections 3.4 and 3.5 .

To examine the reducibility of the measured $I_{\mathrm{c}^{-}}$and $n$-values with the obtained values of $(1-f)(L / s)^{1 / n_{0}}$ and $R_{\mathrm{t}}$, the $V-I$ curves of sections and samples at each $\sigma_{\mathrm{T}}$ were back-calculated by eqs. (2) and (3) with the obtained values. Then, from each calculated $V-I$ curve, the $I_{\mathrm{c}^{-}}$and $n$-values were estimated. Thus obtained calculation-based $I_{\mathrm{c}^{-}}$and $n$-values, together with experimental results, are shown in Fig. 6. The experimental results were reproduced satisfactorily, suggesting that the $I_{\mathrm{c}^{-}}$and $n$-values of both sections and sample, and the correlation between $n$-value and critical current both for sections and sample, could be accounted for systematically from the obtained values of $(1-f)(L / s)^{1 / n_{0}}$ and $R_{\mathrm{t}}$. This suggestion will be realized in Subsection 3.5.
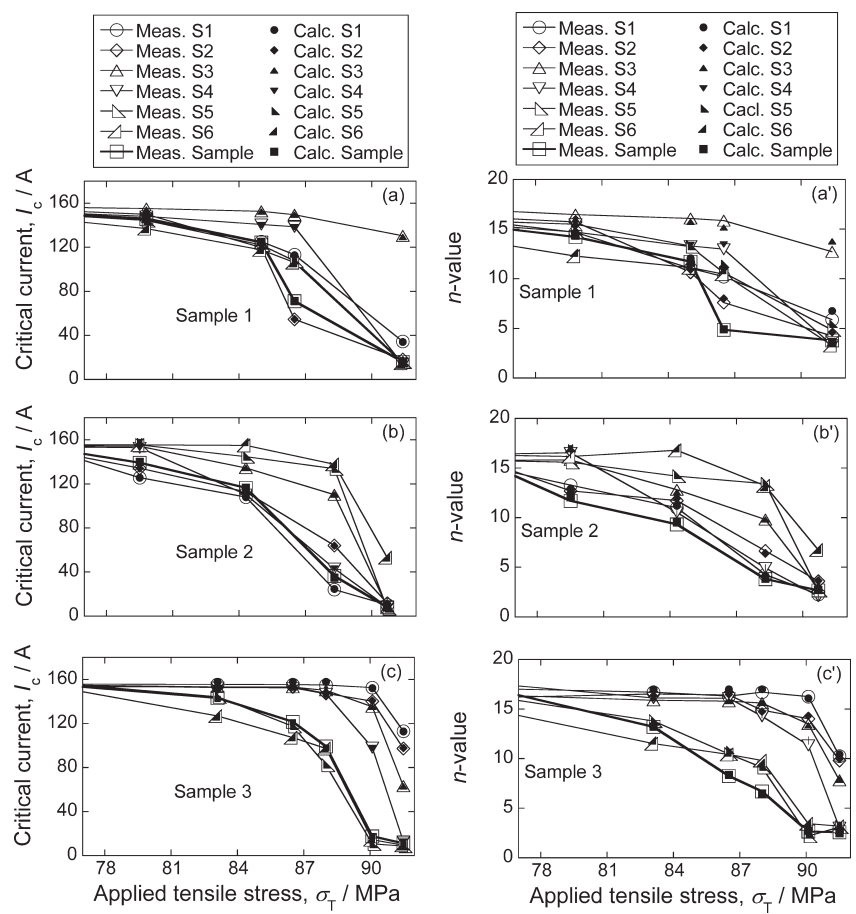

Fig. 6 Comparison of the measured (a, b, c) critical current $I_{\mathrm{c}}$ and (a', b', c') $n$-value of the sections and samples with the calculated values.

\subsection{Influence of the position relation of $V-I$ curve among sections on $I_{c}, n$-value and $R_{t}$ of sample}

To account for the influence of the position relation of $V-I$ curve among the sections on $I_{\mathrm{C}^{-}}$and $n$-values and also on $R_{\mathrm{t}}$ of sample, two examples (Example A and Example B) were picked up from the experimental results and analyzed with an equivalent crack-current shunting model. Example A was taken from the results of Sample 1 at $\sigma_{\mathrm{T}}=85.0 \mathrm{MPa}$ (Fig. 2(c)) and Example B from those of Sample 3 at $\sigma_{\mathrm{T}}=86.5 \mathrm{MPa}$ (Fig. 2(c')). The measured $V-I$ curves of sections and samples in both examples were converted to $E$ (electric field = $V / L)-I$ (current) curves, as shown in Fig. 7(a) and (a'), respectively. The $I_{\mathrm{c}^{-}}$and $n$-values of each section (S1 to S6) and each sample in Examples A and B are shown in Fig. 7(b) and (b'), respectively. The $I_{\mathrm{c}}$-value of sample in Example A $\left(I_{\mathrm{c}}=\right.$ $123 \mathrm{~A})$ was almost the same as that in Example B $\left(I_{\mathrm{c}}=122 \mathrm{~A}\right)$ but $n$-value was evidently different between Examples A ( $n=$ $11.7)$ and $\mathrm{B}(n=8.3)$, as shown in Fig. 7(b) and (b'). It is noted that the $I_{\mathrm{c}}$-value of the sample was within the range between the lowest and highest $I_{\mathrm{c}}$-values of the sections in both examples but $n$-value was different; the $n$-value of sample was lower than the lowest $n$-value among the sections in Example $\mathrm{B}$, while it was in the range between the lowest and highest $n$-values of sections in Example A.

As stated in Subsection 3.2, for both sections and samples, $n$-value was estimated as the index in the form of $E \propto I^{\mathrm{n}}$ in the range of $E=0.1 \sim 10 \mu \mathrm{V} / \mathrm{cm}$. In Example A, the $V-I$ curves of sections were dense in the neighborhood of the $V-I$ curve of the most extensively cracked section with the lowest $I_{\mathrm{c}}$-value among the section. Therefore, the $V-I$ curves of many sections contributed to construct the $V-I$ curve of sample (Fig. 2(c)). This feature is reflected in Fig. 7(a) where the $E$ of the $E-I$ curve of sample went up with $I$ similarly to that of sections in the neighborhood. Accordingly, the $n$-value of 

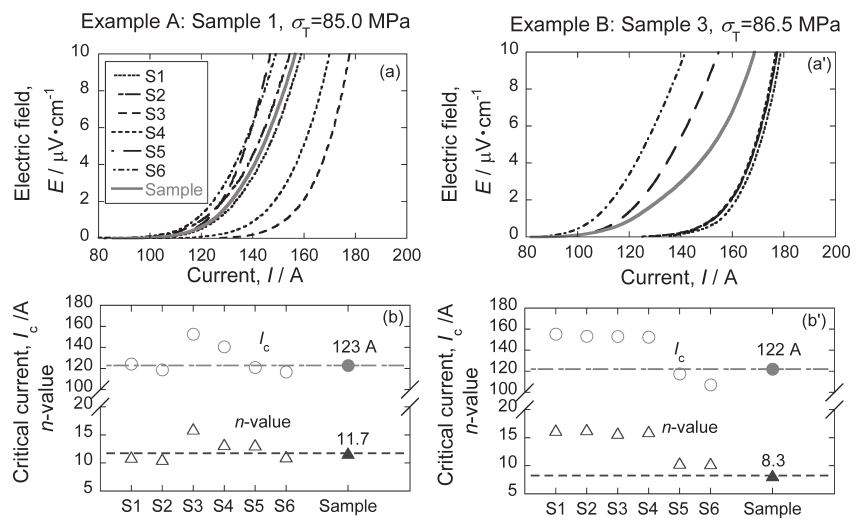

Fig. 7 Measured (a, a') E(electric field) $-I$ (current) curves and (b, b') critical current $I_{\mathrm{c}}$ and $n$-value of the sections and samples in Example A $(\mathrm{a}, \mathrm{b})$ and Example B (a', b'). These examples were taken up due to the feature that the critical current $I_{\mathrm{c}}$ was similar to each other (A:123 A, B:122 A, difference $0.08 \%$ ) but $n$-value was largely different (A:11.7, B:8.3, difference $29 \%$ ) due to the difference in position relation of the $V-I$ curves between the most extensively cracked section and the other sections.

sample was similar to the $n$-values of sections. Actually the $n$-value of sample was in the range of the highest and lowest $n$-values of sections (Fig. 7(b)). On the other hand, in Example $\mathrm{B}$, the $V-I$ curves of only a few sections contributed to construct the $V-I$ curve of sample (Fig. 2(c')), and hence the $E$ of the $E-I$ curve of sample went up slowly with $I$ in comparison with that of sections (Fig. 7(a')). Accordingly, $n$-value of sample was lower than all $n$-values of sections (Fig. 7(b')).

In this way, even when the $I_{\mathrm{c}}$-values are the same, different $n$-values can exist in samples due to the difference in position relation of the $V-I$ curves between the most extensively cracked section and the other sections, which differs from sample to sample. From the analysis of the $V-I$ curves in Fig. 2(c, c'), the values of $R_{\mathrm{t}}$ of the samples in Examples $\mathrm{A}$ and B were estimated to be 2.85 and $0.90 \mu \Omega$, corresponding to high (11.7) and low (8.3) $n$-values, respectively. In this way, the difference in $n$-value between Examples $\mathrm{A}$ and $\mathrm{B}$ with almost the same $I_{\mathrm{c}}$ was described by the difference in $R_{\mathrm{t}}$-value arising from the difference in the position relation of the $V-I$ curves between the most extensively cracked section and the other sections.

\subsection{Influences of voltage probe-distance $L$ on the change in $I_{c}$ and $n$-value with ligament parameter and on the correlation between $\boldsymbol{n}$ and $\boldsymbol{I}_{\mathrm{c}}$}

Figure 8 shows the measured (a, a') $I_{\mathrm{c}^{-}}$and (b, b') $n$-values of sections (voltage probe-distance $L=1 \mathrm{~cm}$ ) and samples $(L=6 \mathrm{~cm})$, plotted against the ligament parameter $(1-f)(L / s)^{1 / n_{0}}$. The solid linear lines in Fig. 8(a, a') refer to the $I_{\mathrm{c}}-(1-f)(L / s)^{1 / n_{0}}$ relation under a virtual case where voltage is developed only at the crack and no current shunting takes place. The measured $I_{\mathrm{c}}$-values were slightly higher than the solid lines due to the occurrence of current shunting in actual test-pieces but were near to them since shunting current is low at low voltage ${ }^{3,4,12)}$. In this way, $I_{\mathrm{c}}$ was, as a first approximation, expressed by $I_{\mathrm{c}} \cong I_{\mathrm{c} 0}(1-f)(L / s)^{1 / n_{0}}$. Namely the ligament size and hence crack size plays a dominant role in determination of $I_{\mathrm{c}}$-value. This feature is common both in filamentary- and coated- tapes.
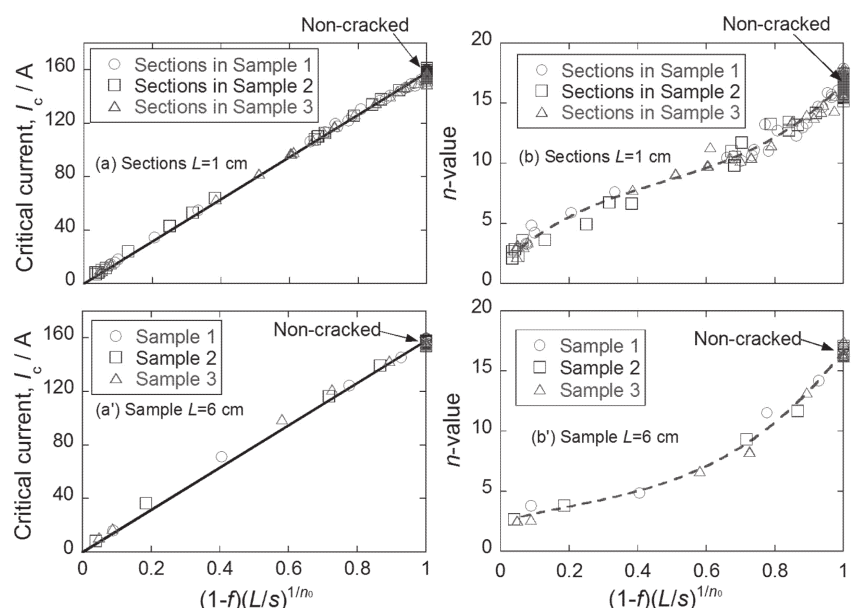

Fig. 8 Measured values of (a, a') critical current $I_{\mathrm{c}}$ and (b, b') $n$-value of sections and samples, plotted against $(1-f)(L / s)^{1 / n_{0}}$. The solid linear lines in $(\mathrm{a}, \mathrm{a})$ refer to the $I_{\mathrm{c}}-I_{\mathrm{c} 0}(1-f)(L / s)^{1 / n_{0}}$ relation under a virtual case where voltage is developed only at the crack and also no current shunting takes place $\left(V_{\mathrm{c}} / R_{\mathrm{t}}=0\right.$ in eq. (5)).

The broken curves in Fig. 8(b, b') show the fitting curves to the experimental results. While $I_{\mathrm{c}}-(1-f)(L / s)^{1 / n_{0}}$ relation was linear as a first approximation for both of samples and sections, $n-(1-f)(L / s)^{1 / n_{0}}$ relation was not linear but was curved, and, in addition, the shape of the $n-(1-f)(L / s)^{1 / n_{0}}$ curve of sections was different from that of samples; $n$-value of samples decreased more significantly with decreasing $(1-f)(L / s)^{1 / n_{0}}$. In this way, the $n$-value decreased with increasing crack size more severely than $I_{\mathrm{c}}$-value when $L$ was larger. These results indicate that, for a given ligament size (namely for a given crack size), $n$-value decreases with increasing $L$ more than $I_{\mathrm{c}}$-value. This indication was clearly found as the difference in the relation of $n$-value to $I_{\mathrm{c}}$ between the sections $(L=1 \mathrm{~cm})$ and samples $(L=6 \mathrm{~cm})$ in Fig. 9; the level of the $n$-value in the $n-I_{\mathrm{c}}$ relation for samples $(L=6 \mathrm{~cm})$ was lower than that for sections $(L=1 \mathrm{~cm})$.

The experimentally obtained $n-I_{\mathrm{c}}$ relation for sections and samples in Fig. 9 were described by the broken and dotted curves, respectively, calculated by eqs. (2) and (3) with $R_{\mathrm{t}}=$ $0.41 \mu \Omega$ and $1.6 \mu \Omega$, respectively. These $R_{\mathrm{t}}$ values were estimated as to give the curves that go through the center of the experimental data points in Fig. 9, and hence they were around the average of $R_{\mathrm{t}}$-values for sections and samples shown in Fig. 5(a', b', c'). If we assume that the sample is constituted of a series of 6 sections having the same $n-I_{\mathrm{c}}$ curve as the broken curve in Fig. 9, the $R_{\mathrm{t}}$-value of the sample is the sum of the $R_{\mathrm{t}}$-values of sections $(0.41 \mu \Omega \times 6=2.46 \mu \Omega)$. Namely, in such an assumed case, the $n-I_{\mathrm{c}}$ curve of the $6 \mathrm{~cm}$-sample for $R_{\mathrm{t}}=2.46 \mu \Omega$ is equal to that of $1 \mathrm{~cm}$-sections for $R_{\mathrm{t}}=0.41 \mu \Omega$. However, the $R_{\mathrm{t}}$-value of $1.6 \mu \Omega$ that can describe the measured $n-I_{\mathrm{c}}$ relation of sample was lower than $2.46 \mu \Omega$ in the assumed case. The reason for this is attributed to the difference in extent of cracking among sections. As the sample is constituted of a series electric circuit of sections, the most extensively cracked section and the sections whose $V-I$ curves are in the neighborhood of the $V-I$ curve of the most extensively cracked section contribute to the voltage of sample, which act to raise $R_{\mathrm{t}}$-value in comparison with the contribution of a single section. However, the contribution of 


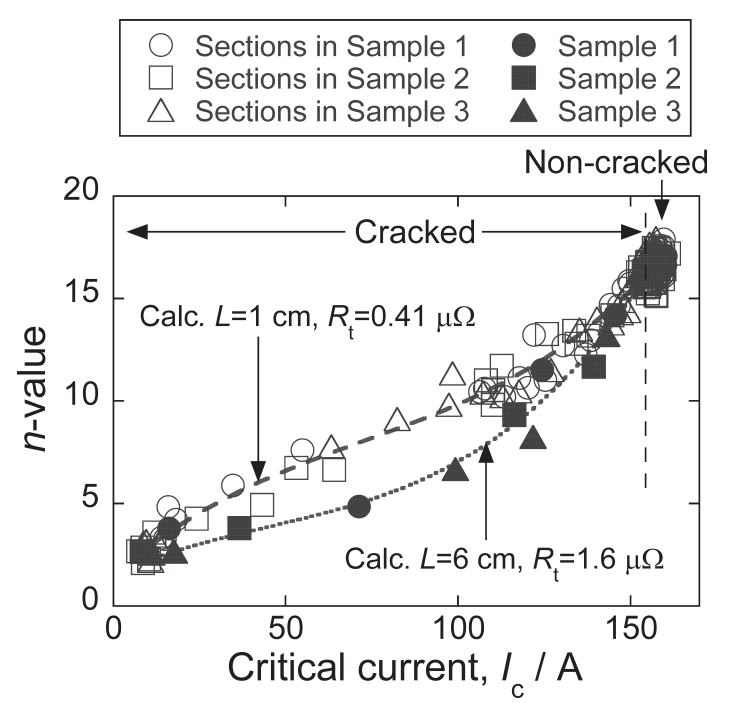

Fig. 9 Measured and calculated correlation between $n$-value and critical current in sections $(L=1 \mathrm{~cm})$ and samples $(L=6 \mathrm{~cm})$, showing the difference in $n-I_{\mathrm{c}}$ correlation between sections and samples. The $n-I_{\mathrm{c}}$ relation curves for sections $(L=1 \mathrm{~cm})$ and samples $(L=6 \mathrm{~cm})$, calculated by eqs. (2) and (3) with $R_{\mathrm{t}}=0.41 \mu \Omega(L=1 \mathrm{~cm})$ and $1.6 \mu \Omega(L=6 \mathrm{~cm})$, are shown with the broken and dotted curves, respectively.

less-cracked-sections to the $V-I$ curve of the sample is small and hence limits the increase in $R_{\mathrm{t}}$-value. Thus the $R_{\mathrm{t}}$-value $(1.6 \mu \Omega)$ of the sample is higher than that $(0.41 \mu \Omega)$ of single section but is lower than the value $(2.46 \mu \Omega)$ in the assumed case. The lower $R_{\mathrm{t}}$-value leads to lower $n$-value. In this way, the tendency for the $n-I_{\mathrm{c}}$ relation to shift to a lower $n$-range with increasing sample length $L$ is described via the change in $R_{\mathrm{t}}$-value with $L$ that reflects the heterogeneous cracking behavior of superconducting filaments.

\section{Conclusions}

(1) While $I_{\mathrm{c}^{-}}$and $n$-values of sections and samples were commonly reduced with increasing applied stress due to the enhanced crack evolution, the extent of reduction of $I_{\mathrm{c}}$ and $n$-value was different among sections and among samples due to the difference in crack evolution behavior even at the same stress.

(2) The most extensively cracked section, whose $V-I$ curve was nearest to that of the sample, played a dominant role in determination of critical current $I_{\mathrm{c}}$ of the sample in comparison with the other sections. On the other hand, $n$-value of the sample was strongly affected not only by the $V-I$ curve of the most extensively cracked section but also by the difference in position relation of the $V-I$ curves between the most extensively cracked section and the other sections; the smaller the difference, the larger became the $n$-value of the sample.

(3) When the difference in crack evolution among the sections was small, the voltages developed in many sections contributed to the voltage of sample. In such a case, $I_{\mathrm{c}}$ and $n$-val- ue of sample were within the range of the highest and lowest values among the sections.

(4) When the cracking in one or two sections was far severer than that of other sections, the voltage developed at the severely cracked sections largely contributed to the voltage of sample. In such a case, $I_{\mathrm{c}}$ of sample was within the range of lowest and highest $I_{\mathrm{c}}$ of the sections, but $n$-value of sample was lower than that of the most severely damaged section.

(5)The measured $I_{\mathrm{c}}$ and $n$-value of both sections and samples were described satisfactorily by an equivalent crack-current shunting model. The measured correlation of $n$-value to $I_{\mathrm{c}}$ both for sections and samples were also described by this model.

\section{REFERENCES}

1) D. Uglietti1, B. Seeber, V. Abächerli, W.L. Carter and R. Flükiger: Supercond. Sci. Technol. 19 (2006) 869-872.

2) K. Osamura, M. Sugano, S. Machiya, H. Adachi, S. Ochiai and M. Sato: Supercond. Sci. Technol. 22 (2009) 065001.

3) S. Ochiai, T. Arai, A. Toda, H. Okuda, M. Sugano, K. Osamura and W. Prusseit: J. Appl. Phys. 108 (2010) 063905.

4) S. Ochiai, H. Okuda, T. Arai, S. Nagano, M. Sugano and W. Prusseit: Cryogenics 51 (2011) 584-590.

5) S. Ochiai, H. Okuda and N. Fujii: Mater. Trans. 55 (2014) 1479-1487.

6) S. Ochiai, H. Okuda, N. Fujii and K. Osamura: Mater. Trans. 56 (2015) 381-388.

7) H.-S. Shin, J. Marlon, H. Dedicatoria, H.-S. Kim, N.-J. Lee, H.-S. Ha and S.-S. Oh: IEEE Trans. Appl. Supercond. 21 (2011) 2997-3000.

8) S. Ochiai, H. Okuda, M. Sugano, S.-S. Oh and H.-S. Ha: Mater. Trans 55 (2014) 549-555.

9) H. Oguro, T. Suwa, T. Suzuki, S. Awaji, K. Watanabe, M. Sugano, S. Machiya, M. Sato, T. Koganezawa, T. Machi, M. Yoshizumi and T. Izumi: IEEE Trans. Appl. Supercond. 23 (2013) 8400304.

10) S. Ochiai, M. Fujimoto, H. Okuda, S.S. Oh and D.W. Ha: J. Appl. Phys. 105 (2009) 063912.

11) S. Ochiai, M. Fujimoto, J.K. Shin, H. Okuda, S.S. Oh and D.W. Ha: J. Appl. Phys. 106 (2009) 103916.

12) S. Ochiai, H. Okuda, M. Sugano, K. Osamura, A. Otto and A.P. Malozemoff: Mater. Trans. 53 (2012) 1549-1555.

13) Y. Miyoshi, E.P.A. Van Lanen, M.M. Dhallé and N. Nijhuits: Supercond. Sci. Technol. 22 (2009) 085009.

14) N. Banno, D. Uglietti, B. Seeber, T. Takeuchi and R. Flükiger: Supercond. Sci. Technol. 18 (2005) 284-288.

15) H. Kitaguchi, H. Kumakura and K. Togano: Physica C 363 (2001) 198-201.

16) H. Kitaguchi, A. Matsumoto, H. Hatakeyama and H. Kumakura: Physica C 401 (2004) 246-250.

17) S. Ochiai, H. Okuda, M. Fujimoto and K. Osamura: Mater. Trans. 56 (2015) 1558-1564

18) A. Otto, E.J. Harley and R. Marson: Supercond. Sci. Technol. 18 (2005) S308-S312.

19) S. Ochiai, H. Matsubayashi, H. Okuda, K. Osamura, A. Otto and A. Malozemoff: Supercond. Sci. Technol. 22 (2009) 095012.

20) S. Ochiai, T. Nagai, H. Okuda, S.-S. Oh, M. Hojo, M. Tanaka, M. Sugano and K. Osamura: Supercond. Sci. Technol. 16 (2003) 988-994.

21) A. Kelly and W.R. Tyson: J. Mech. Phys. Solids 13 (1965) 329-350.

22) S. Ochiai: Mechanical Properties of Metallic Composites (Ed. Ochiai S) Marcel Dekker Inc., New York, (1993) 473-510.

23) S. Ochiai and M. Hojo: J. Mater. Sci. 31 (1996) 3861-3869.

24) Y. Fang, S. Danyluk and M.T. Lanagan: Cryogenics 36 (1996) 957962. 